\title{
Characterization of novel rounder aromatic Isatin ester anti human immunodeficiency virus
}

\begin{abstract}
We report here eight novel rounder aromatic isatin ester anti-human immunodeficiency viruses. One among those is a more bioactive and related factors. It has shown that information related to the molecular shape correlates and modified with activity nitro aromatic ester compound with a rounder rather than a more linear compound.
\end{abstract}

Keywords: nevirapine; Efavirenz; rilpivirine; etravirine; molecular database

\author{
Volume 2 Issue 3 - 2018
}

\author{
Rahul Hajare \\ Department of National AIDS Research Institute,Vinayaka \\ Mission University, India
}

Correspondence: Rahul Hajare, Department of National AIDS Research Institute,Vinayaka Mission University, India, Email rahulhajare@rediffmail.com

Received: March 30, 2018 | Published: May 10, 2018

\section{Introduction}

A human immunodeficiency virus strains has sloppy in its reproduction. However, due to the infidelity of HIV-1 during replication, the emergences of mutations (especially K103N and Y181C) rapidly develop resistance and high cytotoxicity rate to the first- generation with rigid structures, such as Nevirapine (NVP) and Efavirenz (EFV). ${ }^{1,2}$ Representative second generation improved drug resistance profiles have recently marketed diarylpyrimidine analogues rilpivirine (TMC278, RPV) and etravirine (TMC125, ETR). ${ }^{3,4}$ Researcher has looked about untapped chemical space in inhibitors binding pocket, also explore unresolved chemical modification obtaining more potent, quality, efficacy and safety back-up extraordinary series of unique rounder aromatic Isatin ester connected by flexible linkers have been investigated. Supporting information has collected from virtual laboratory HIV drug resistance database Stanford University). ${ }^{5,6}$

\section{Compounds and reagents}

Compounds have authorized from Sigma- Aldrich Company (MO, USA) and structure compounds, HIV activity has amplified using special brightlite reagent. Agilent Cary 630 FTIR spectrometer, Luminometer for virus counts and ELISA analyzer.

\section{Cells and viruses}

Engineered TZM-bl cell line has provided by the TZM-bl NIH AIDS Reagent Program USA. This has indicator cell line. Laboratory adapted strains, including HIV-1UG070, 7th PID dated 05/12/14 has obtained from the Department of Molecular Virology, Biosafety Laboratory-II NARI Pune.

\section{Interpretation of gold dock}

A computer program designed to screen rapidly by performed using Gold Dock Suite. The coordinates for Human Immunodeficiency Virus -I 3M8Q and Shanghai small molecular database (SSMD) has been utilized. Breakpoint and RMS breakthrough highly greater efficacy of compound. Insertion of bridge to K101 active. The computational simulation of Etravirine, next generation drug. In docking study standard Etravirine [a] and target compound with K101 residue because the second position brought. In this context $\mathrm{C}=\mathrm{O}$ is a oxygen isolated in the active compound aromatic Isatin Ester benzene sulfa methaxazole they formed hydrogen bond with back bone of K101 residue which has crucial for drug resistance and thus compromised the untapped chemical space in IBP and this increase the affinity of compound toward strain inhibition. It is further conclude that region having $\mathrm{OCH}_{3}, \mathrm{Br}, \mathrm{CH}_{3}, \mathrm{Cl}$ of $\mathrm{C}-5$ and distance between interaction 2.44 and combined K103, prone to mutation at the IBP entrance. Like pyrrole ring substitution on $\mathrm{C}-1$ by morpholine showed higher activity as it has made vander walls force that are acting upon TYR 188 with distance 3.307 between residue atom and ligand atom which going to stabilized the inhibitor binding pocket and it should be prevent the inhibitor from leaving the binding pocket. Vanderwalls force interaction act on TYR 188 which made flexible clamp, however mutation resulting in looses or any changes interaction. Most important in this research and finding substitution at $\mathrm{C}-3$ by sulphonamide based substituents may be lead pi-pi stacking interaction between compound and aromatic amino acid residue TYR 181 with interaction distance 5.9 which is prone to mutation resulting loss or change of interaction Figure 1. This clearly indicates that the inhibitor binding process and residues must facilitate to enter the binding pocket and adopt an appropriate conformation is the optimal interaction with the surrounding residue. This lead to change due to conformation of $U$, shoes horse like structure, made sites feedback inhibitor to regulate the activities and showed higher activity. ${ }^{8,9}$ Interaction for instance 3M8Q (Resistance mutant virus) and Reference ligand. Covalent bonds to protein have not displayed. Image generated using pose view software. By downloading this image confirmed to follow citation rule. 3D structure of the docked conformation of novel aromatic Isatin Ester (1C) and BP of K103N/Y181C mutant HIV- 1 strain, (PDB code:3M8Q), showed interaction of target compound in purple colour and 3M8Q in green and blue colour. Predicted figure showed the hydrogen bonding with K101 residue, hydrophobic interaction, pi-pi interaction, hydrogen bonds formed between target compound and K101 has maintained which can be crucial for drug resistance and merged molecule insertions position in inhibitor-binding pocket (IBP) (PDB:3M8Q) cavity endorsed lock form in open position and stop the transcription. 


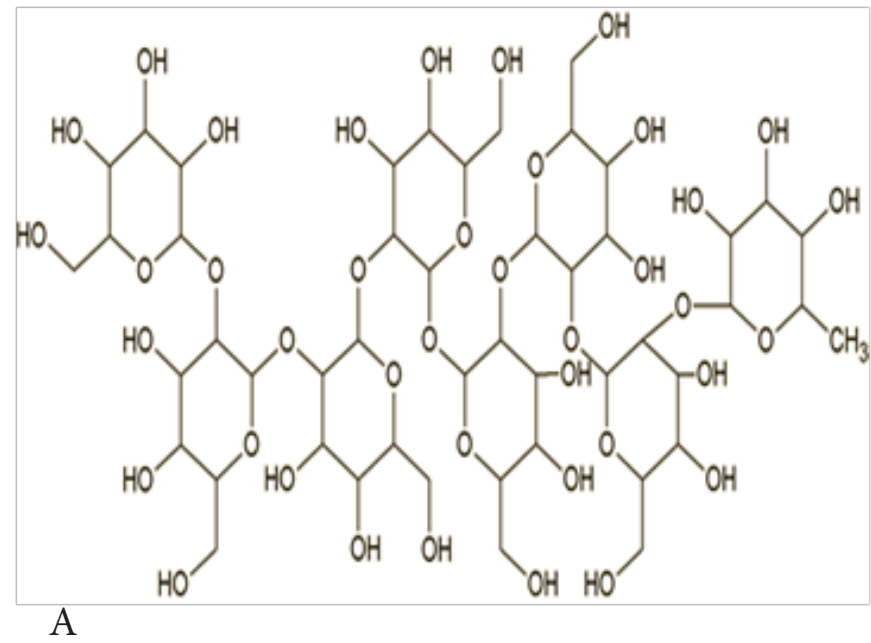<smiles>CCCCCCCCCCCCCCCCCC(=O)OC1C(OC2C(OC3C(OC4C(Cc5ccc(C(=O)O)cc5OC)OCC(O)C4O)CCC(O)C3O)OCC(O)C2O)OCC(O)C1O</smiles>

B

Figure I Carbon content produce black dashed line-hydrogen bond, salt bridge, metal interaction, green solid line-hydrophobic interaction, green dashed line-pi-pi, cation-pi interaction.

\section{Conclusion}

A comparison of the designed, synthesized and evaluated rounder novel aromatic ester which showed nanomolar activity against HIV1. Tasted series of aromatic Isatin Ester possess inhibitory activity displays potency (IC50 at micromolar level) detection UG070 7 th PID (refence protein 3M8Q PDB). At best, binding energy could be related to IC50 in assay, more negative of the binding energy results in the formation of stronger complexes belonging therefore when a ligand have a low binding energy its affinity towards the HIV-1 target has bigger target compound adaptable. The detection of binding energy value of refence protein and reference ligand complexes shows $-12.43 \mathrm{kcal} / \mathrm{mol}$ and average of experimental binding energy values of new ligands and refence protein complexes were shows $11.60 \mathrm{kcal} / \mathrm{mol}$. Target compound aromatic Isatin Ester benzene sulfa methaxazole has been shown binding energy $-8.69 \mathrm{kcal} / \mathrm{mol}$, which has showed its affinity towards the protein 3M8Q has sizeable in comparison with other lead ligands with higher binding energy. IC50 of target compound aromatic Isatin Ester benzene sulfa methaxazole has found to be $4.91 \mu \mathrm{g} / \mathrm{ml}$. Also it has been clearly indicates strain- target compound inhibitor binding energy values highly correlated which has less than to reported determined binding energy values, which has high degree to cover all edges and outskirt in IBP and reduce the frequency of chemical space creation. The differences in these correlations may reflect good ground of biological features of the interactions of HIV-1 inhibitor and its complexes. A thorough study has carried out over twenty computationally designed aromatic Isatin ester analogs using gold dock suite and program with the goal of identifying potential lead molecules that bind to the HIV-1 mutant protein respectively. The comparatively higher interaction scores of lead compounds compared to Etravirine when docked with HIV-1 mutant protein at the IBP active site residue suggest these novel leads would potentially bind more strongly to the pockets of HIV-13M8Q proteins. Further, the leads have docked with Lys101, Val106, Leu234 and His 235 this proteins residue to predict their binding efficiencies with HIV-1 protein. All the twenty designed aromatic Isatin ester and its analogs with chemical substitutions at the $\mathrm{X} 1, \mathrm{X} 3, \mathrm{X} 5 \rightarrow$ rounder showing better interactive scores respectively than reference ligand ( Interactive score $=-152.57$ ), as a result of docking of among twenty analogs eight lead have showed interactive score $-81.69,-88.28$, $-80.98,-55.92,-85.35,-74.04,-67.87$ and -78.17 and in vitro experiment IC 50 has found $25.65 \mu \mathrm{g} / \mathrm{ml}, 31.18 \mu \mathrm{g} / \mathrm{ml}, 4.91 \mu \mathrm{g} / \mathrm{ml}$, $32.01 \mu \mathrm{g} / \mathrm{ml}, 4.59 \mu \mathrm{g} / \mathrm{ml}, 3.96 \mu \mathrm{g} / \mathrm{ml}, 5.02 \mu \mathrm{g} / \mathrm{ml}$ and $25.34 \mu \mathrm{g} / \mathrm{ml}$. To date the target molecule $1 \mathrm{C}$, when docked with $3 \mathrm{M} 8 \mathrm{Q}$, have shown better dock scores (Interaction Score - 80.98 compared to reference ligand (Interaction Score - 152.57). Hence these have expected to bind strongly onto inhibitor binding pockets and highly functional lead $7.15 \mathrm{ug} / \mathrm{ml}$ to cell lines. TZM- bl and displayed potent anti- HIV1 activity found $4.91 \mu \mathrm{g} / \mathrm{ml}$ against laboratory adapted strains UG070, 7 thPID.

\section{Acknowledgments}

I would like to express deep gratitude to my post- doctoral guide Renowned Scientist, Respected Dr. Ramesh S. Paranjape (Retired Director \& Scientist 'G' National AIDS Research Institute, India.

\section{Conflict of interest}

Ethics statement. Principal and study of the Biosafety Laboratory II Biosafety Laboratory III, National AIDS Research Institute (ICMR) Pune. This processes Indian Council of Medical Research Government of India.

\section{References}

1. Paranjape R, Hajare R. A Mechanistic Study of Anti-HIV Activity and Cancer Registry Match to Laboratory Drug 2 Indolinones Shows Record Linkage among Them Many Interesting Lead Evaluation. HIV Curr Res. 2018;(3):1-126.

2. Paranjape R, Hajare Rahul. Association between Technology Development and Rounder Substituted-1h-Indole-2,3-Dione Hiv-1 Inhibitors Who Have Displays Strategic Nanomolar Cytotoxicity. Organic \& Medicinal Chem IJ. 2018;6(3):555686.

3. Paranjape R, Hajare Rahul. Generation and Characterization of New Entrance Antiretroviral Drug 2-Indolinone: Results of a Classical R \& D Study. Organic \& Medicinal Chem IJ. 2018;6(2):555685.

4. Rahul H, Ramesh P, Smita K. Tailored microwave technology for Synthesis N'-[(3Z)-5-chloro-1-(morpholin-4-ylmethyl)-2-oxo-1,2dihydro-3H-indol-3 ylidene] pyridine-4 carbohydrazide as HIV-1 inhibitors. Drug Formulation \& Bioavailability Congress. 2016. 
5. Rahul H, Ramesh P, Smita K. Technology development and design of novel 1, 3, 5-tri substituted-1H-indole-2,3-dione: HIV-1 Inhibitors with displays strategic nanomolar cytotoxicity. USA; 2016.

6. Rahul H, Smita K, Madhuri T, et al. Variability interaction between Etravirine and Rilpivirine: a Retrospective. KJACT. 2016;2(1):100103.

7. Rahul H, Smita K, Madhuri T, et al. Technology Development and Design of Novel 1, 3, 5-tri Substituted-1H-Indole-2, 3-Dione HIV-1 Inhibitors With Displays Strategic Nanomolar Cytotoxicity. World J Pharm Pharm Sci. 2016;5(6):1-391.
8. Rahul H, Smita K, Madhuri T, et al. Isatin Anti-HIV Agent: A Review. World journal of PAPS. 2016;5(7):569-575.

9. Rahul AH, Smita S, Kulkarni R, et al. Design Space Filling Model, Synthesis and Evaluation of Novel 2-Indolinone HIV-1 Inhibitors. International Journal of Advanced Research. 2015;3(12):1332-1335. 